\title{
APROXIMAGIÓN A LA ENSEÑANZA DE LAS SUCESIONES DE NÚMEROS REALES POR MEDIO DE LOS ESPACIOS DE TRABAJO MATEMÁTICO
}

\author{
APPROACH TO THE TEACHING OF SEQUENCES OF REAL NUMBERS \\ BY MEANS OF MATHEMATICAL WORKING SPACES
}

Paula Verdugo Hernández, paulasinttia@gmail.com

Universidad Adventista de Chile, Chillán, Chile

\section{RESUMEN}

Este artículo tiene como objetivo principal investigar el trabajo matemático de profesores universitarios respecto de la enseñanza de las sucesiones de números reales (sucesiones) en el aula. Mediante la teoría de los Espacios de Trabajo Matemático (ETM) analizamos algunos elementos de la enseñanza (ETM idóneo) de este objeto, tomando en cuenta las componentes del plano epistemológico y cognitivo, identificando las génesis, así como también los paradigmas del análisis real. Concretamente, se presenta un estudio de caso sobre el ETM idóneo de tres profesores universitarios, solicitándoles realizar una producción donde aborden la enseñanza de la sucesión $\left(a_{n}\right) n \in \mathrm{N}$ definida por $a_{n}=(1+1 / n)^{n}$ para cada $n \in \mathrm{N}$. Los principales resultados evidencian diferencias entre los ETM idóneos, observándose que estos se enmarcan dentro de distintos paradigmas del análisis real, activando distintas componentes del ETM. Por último, en base a lo anterior, sugerimos algunos elementos que harían factible la estructuración de un ETM idóneo más completo para las sucesiones.

\section{PALABRAS CLAVE:}

ETM idóneo; enseñanza de las sucesiones; enseñanza

universitaria; paradigmas del análisis real.

\section{ABSTRACT}

The main goal of this article is to investigate the mathematical work of university professors regarding the teaching of real number sequences (sequences) in the classroom. Through the Mathematical Working Spaces (MWS) theory, we analyze some elements of the suitable MWS of this object, taking into account the components of the epistemological and cognitive aspects, identifying the geneses, as well as the paradigms of the real analysis. Specifically, a case study on the suitable MWS of three university professors is presented, whom we asked to tackle the teaching of the sequence $\left(a_{n}\right) n \in \mathrm{N}$ defined by $a=(1+1 / n)$ for each $n \in \mathrm{N}$. The main results show differences between the suitable MWS, observing that they are framed within the distinct paradigms of real analysis, activating different components of MWS. Finally, based on these results, we suggest some elements that would make feasible the structuration of a more complete suitable MWS for the sequences.

\section{KEYWORDS:}

Suitable MWS; real sequences teaching; university

teaching; paradigms of real analysis. 


\section{Introducción}

Este trabajo tiene como objetivo principal estudiar parte del ETM idóneo de las sucesiones reales en la enseñanza universitaria. Es importante señalar que las sucesiones reales constituyen un tema de estudio interesante, debido a la presencia de la noción de convergencia, concepto fundamental en las matemáticas. Existen diversos estudios que se han dedicado a tratar este objeto matemático desde el punto de vista didáctico y/o educativo; entre ellos, el trabajo realizado por Robert (1982), el cual estudia y clasifica en modelos a las diferentes formas de conceptualización de las descripciones de los estudiantes sobre la convergencia de sucesiones numéricas por intermedio de discursos, ejemplos y representaciones. En dicho trabajo se destaca el modelo dinámico, en donde la convergencia es asociada a la idea de aproximación; el modelo estático, el cual traduce al lenguaje natural la definición $(\varepsilon, N)$ de la convergencia de sucesiones, y el modelo monótono, en el que la distancia entre los términos de una sucesión y su límite decrece, lo cual constituye un caso particular. Para estudiar los modelos anteriores, la autora diseñó y aplicó un cuestionario a estudiantes de enseñanza media en Francia. Los resultados de los estudiantes marcan ciertas diferencias: aquellos que tenían un modelo estático contestaron correctamente, aquellos con un modelo monótono respondieron incorrectamente $y$, por último, de los que tenían un modelo dinámico, la mitad respondió adecuadamente, mientras que la otra mitad respondió erróneamente.

Por otro lado, Bloch (2000), en el capítulo 6 de su tesis doctoral, desarrolla e implementa una situación para el aprendizaje de la noción de convergencia de sucesiones, dirigida a nivel secundario, en la cual se solicita al estudiante calcular los términos generales de la sucesión de perímetros y áreas de los copos de nieve de Von Koch, así como estudiar su convergencia. Para ello, el docente indica a los estudiantes ciertos criterios de validación de la convergencia, los cuales no corresponden a la noción rigurosa. La autora asevera que la situación se desarrolló dentro de lo estipulado: los estudiantes lograron darse cuenta de que la sucesión de perímetros era creciente y no acotada, por lo tanto, divergente. Asimismo, notaron que la sucesión de áreas, si bien era creciente, era acotada, y lograron calcular el límite observando la tendencia de los términos de la sucesión mediante calculadora (criterio de exploración). Los criterios de exploración y validación se pudieron implementar, los estudiantes los adaptaron para encontrar el correspondiente para una sucesión convergiendo a 0. Asimismo, la situación permitió institucionalizar una definición correcta de una sucesión convergente al infinito y de una convergente a $\mathrm{O}$.

Oktaç y Vivier (2016) señalan que la noción de límite ha sido estudiada en educación matemática por cerca de 40 años, incluyendo límites de sucesiones y funciones. Según estos autores, las razones por las cuales esta noción ha sido tan estudiada son, por una parte, que ella presenta grandes dificultades para el aprendizaje de los estudiantes, y por otra, que la noción de límite, siendo una noción fundamental del análisis, se relaciona con varias otras nociones tales como continuidad, diferenciabilidad, etc., de modo que las dificultades asociadas a los límites podrían también tener un impacto en el aprendizaje de tales conceptos.

Por su parte, Mamona-Downs (2001) presenta una secuencia didáctica de convergencia de sucesiones con el fin de resolver los problemas de comprensión ligados al estilo minimalista de la noción de convergencia de sucesiones, esto es, el hecho de que la proposición que define la convergencia es muy compacta, llena de cuantificadores, y al mismo tiempo no se dice que el épsilon sea pequeño, ni que el $\mathrm{N}$ dependa de épsilon, es decir, en la definición está implícito el rol de las variables involucradas, lo cual no se señala directamente. Todo lo anterior requiere una reflexión madura sobre su estructura, sobre el contenido mismo de la definición, fijándose en sus aspectos metacognitivos y cognitivos de su diseño.

Asimismo, las sucesiones no solamente son importantes por su relación con la convergencia, sino que también por el hecho de que existen diversas aplicaciones de estas en las ciencias y la ingeniería, puesto que ellas participan en procesos de aproximación numérica. Debido a lo anterior, las sucesiones forman parte de los programas de estudio para la formación de ingenieros, y específicamente son estudiadas en los cursos de cálculo de primer o segundo año de universidad en dichas carreras.

El estudio realizado por Verdugo-Hernández (2018) da cuenta de la riqueza epistemológica de las sucesiones reales. En efecto, los estudios iniciales realizados por Cauchy (1789-1857) fueron imprescindibles para el desarrollo del análisis real y en particular de las sucesiones (Dhombres, 1980). Asimismo, los trabajos desarrollados por Weierstrass (1815-1897) y sus colaboradores complementan las demostraciones de Cauchy, clarificando las nociones de convergencia uniforme, continuidad uniforme, y derivación e integración término a término de una serie infinita (Hairer y Wanner, 2000), en las cuales las sucesiones juegan un rol clave. Por su parte, Cantor y Dedekind, hacia los años 1872 y 1888, presentan una construcción equivalente de los números reales. Posteriormente, Hilbert desarrolla en 1899 el método axiomático, mediante el cual introduce el sistema de los números reales, que resulta ser un cuerpo conmutativo totalmente ordenado y arquimediano, tal que no es posible agrandarlo de manera de obtener un nuevo sistema numérico que satisfaga dichas propiedades. Este último aspecto constituye la estructuración de aquello que Cantor y Dedekind denominaron "completitud" de los números reales (Dhombres, 1980). La construcción de Cantor se apoya sobre las sucesiones fundamentales, hoy en día conocidas como sucesiones de Cauchy. En efecto, 
Cantor demuestra que todo número real (en particular, todo número irracional) puede ser aproximado por una sucesión de Cauchy de números racionales. De la discusión previa evidenciamos la importancia de las sucesiones, las cuales contribuyeron de manera decisiva a dar fundamento al análisis moderno.

En esta investigación, hemos decidido analizar la enseñanza de la sucesión $\left(a_{n}\right) n \in \mathrm{N}$ definida por

$$
a_{n}=(1+1 / n)^{n} \quad \text { para cada } \quad n \in \mathrm{N}
$$

dicha sucesión posee una presencia importante en el estudio del análisis, siendo un ejemplo recurrente en varios textos universitarios como Kuratowski (1962), Bobadilla y Labarca (2004) y Stewart (2012). Además, constituye un importante ejemplo de aplicación del criterio de convergencia de las sucesiones monótonas y acotadas, tal como se aprecia en algunos textos universitarios como los señalados, que ejemplifican dicho criterio por medio de esta sucesión. Por último, la sucesión mencionada es significativa en las aplicaciones ya que se utiliza para modelizar problemas de interés, entre otros (Stewart, 2012). Por todo lo anteriormente expuesto, la enseñanza de la misma amerita ser estudiada.

En términos de nuestro marco teórico, estudiamos las posibles génesis activadas entre las distintas componentes del plano epistemológico y cognitivo. Asimismo, analizamos los planos verticales activados y los paradigmas en los cuales se posiciona el trabajo matemático. De esta manera, se ha podido examinar similitudes y diferencias en términos del ETM idóneo de tres docentes universitarios, de modo tal de proponer mejoras que converjan hacia la construcción de un posible trabajo matemático completo de las sucesiones, el cual incluya la activación de las tres génesis, junto a los tres planos verticales, al igual que todos los paradigmas. Debido a la amplitud del ETM idóneo, para efectos de este trabajo nos hemos enfocado en el estudio de una parte de este, dispuesto en la enseñanza universitaria de las sucesiones.

A continuación, presentaremos brevemente nuestro marco teórico, el cual nos ha permitido situar nuestra problemática en términos precisos y en particular estudiar en detalle el rol que juegan las distintas componentes del plano epistemológico y cognitivo del Espacio de Trabajo Matemático en las sucesiones. Enseguida, presentamos el diseño metodológico. Consecutivamente, mostramos y discutimos los resultados de los análisis sobre la enseñanza de la sucesión $\left(a_{n}\right) n \in \mathrm{N}$. Finalmente, presentamos las conclusiones de este trabajo.

\section{Espacio de Trabajo Matemático}

El Espacio de Trabajo Matemático (ETM) (Kuzniak, 2011) tiene por objetivo principal estudiar y favorecer el funcionamiento del trabajo matemático en un contexto educativo determinado. Para definir el ETM se introducen dos planos: el plano epistemológico y el cognitivo, que estructuran el ETM apoyando la comprensión tanto del modelo del trabajo matemático que se genera, como también de la articulación entre sus planos mediante tres génesis (Kuzniak, Montoya-Delgadillo y Vivier, 2016): semiótica, instrumental y discursiva. Se identifican tres tipos de ETM (Kuzniak, 2011): ETM de referencia, relativo a criterios matemáticos definidos por una institución, que requiere un ETM idóneo, del cual depende tanto el diseño de la clase como de las tareas encomendadas a los estudiantes, quienes deberán comprometerse en actividades con el fin de realizarlas de acuerdo a su ETM personal. Por lo anterior, según Kuzniak y Richard (2014), el ETM idóneo no está fijado y se debe ajustar a ciertas restricciones locales, que dependen del ETM de referencia.

Adicionalmente el ETM considera, según Kuzniak y Richard (2014), tres planos verticales. Dichos planos actúan sobre la base de ciertas génesis y sus relaciones con el plano epistemológico y cognitivo. El primer plano [Sem-Ins] está sustentado por las génesis semiótica e instrumental. El segundo plano [Sem-Dis] está sustentado bajo las génesis semiótica y discursiva. Por último, el tercer plano [Ins-Dis] se apoya en las génesis instrumental y discursiva, las cuales evidenciaremos en este escrito a través de entrevistas con tres profesores universitarios en ejercicio. Más detalles del modelo del ETM pueden ser encontrados en Gómez-Chacón, Kuzniak y Vivier (2016).

Finalmente, el trabajo matemático está guiado por paradigmas que caracterizan los diferentes ETM. Montoya y Vivier (2016) han propuesto tres paradigmas con el fin de caracterizar el trabajo matemático del análisis real. Ellos fueron identificados teniendo en cuenta la perspectiva histórica y matemática. Tal como se ha discutido en el trabajo de Verdugo-Hernández (2018), y tal como se ha expuesto brevemente en la introducción de este escrito, la completitud de los números reales constituye un aspecto clave en el quehacer del análisis real. En ese sentido, Bergé (2008) identificó la transición del cálculo al análisis como un cambio de trabajo relacionado con la completitud de los números reales. Asimismo, Artigue (1998) identificó una ruptura entre el álgebra y el cálculo con relación al hecho de que en el álgebra, para demostrar una igualdad, se debe realizan una serie de manipulaciones algebraicas, mientras que en el cálculo (o análisis), para demostrar la igualdad entre dos cantidades, basta probar que para todo $\varepsilon>0$, la distancia entre dichas cantidades es menor que $\varepsilon$. Se puede observar que en el análisis se involucra el trabajo con $\varepsilon$, con lo cual implícitamente se está usando la completitud. De acuerdo a lo anterior, el trabajo del análisis involucra este aspecto de manera explícita, lo cual implica necesariamente una reflexión sobre las reglas del cálculo y no solamente su aplicación mecánica para resolver un determinado problema.

Por otro lado, desde la perspectiva histórica, la geometría jugó un rol importante en el desarrollo del análisis. Por ejemplo, Bolzano (1781-1848) planteó la idea de basar el análisis en la geometría, especialmente en la percepción visual de ciertas propiedades tales 
como el Teorema del Valor Intermedio (TVI). Así, viendo el gráfico de una función, es posible determinar si la ecuación $f(x)=0$ posee una solución, lo cual es un caso particular del teorema de Bolzano. Pero, en esta intuición gráfica, implícitamente se está utilizando la completitud de los números reales.

En consecuencia, se han identificado los siguientes paradigmas del análisis real:

Análisis-Geométrico/Aritmético (AG), que permite interpretaciones con hipótesis implícitas basadas en la geometría, el cálculo aritmético o el mundo real.

Análisis-Calculatorio ( $\mathrm{AC})$, donde las reglas de cálculo son definidas más o menos de manera explícita, y se aplican independientemente de la reflexión de la existencia y naturaleza de los objetos introducidos.

Análisis-Real (AR), caracterizado por un trabajo que considera la aproximación y abiertos, incluso lo topológico, y en el que las definiciones y propiedades son establecidas teóricamente permitiendo un "trabajo $\varepsilon^{\prime \prime}$ específico de este paradigma, cotas, desigualdades, "lo despreciable".

Por la naturaleza del objeto matemático considerado, y por el nivel en el cual los docentes enseñan, es esperable que se pueda observar la presencia de varios paradigmas (con mayor fuerza en el paradigma AC) en la resolución de una tarea.

\section{Elementos metodológicos}

El análisis que se presenta en esta investigación es de carácter cualitativo, mostrando un caso con relación al trabajo realizado por tres profesores universitarios, respecto a la sucesión $\left(a_{n}\right) n \in \mathrm{N}$ definida por $a_{n}=(1+1 / n)^{n}$ para cada $n \in \mathrm{N}$.

Con el fin de identificar algunos elementos del ETM idóneo de las sucesiones, se entrevistaron a estos tres profesores de universidad, los cuales serán nombrados como PU1, PU2 y PU3, respectivamente. Algunos antecedentes de los docentes son: PU1 es pedagogo en Matemática, magister en Didáctica de la Matemática, y realiza clases de Cálculo I y II en carreras de Ingeniería Comercial. PU2 es ingeniero civil matemático, magister y doctor en Estadística, y realiza clases en los cursos de Cálculo I, II y Estadística en carreras de Ingeniería Civil. Por último, PU3 es pedagogo en Matemática, magister en Matemática, y realiza clases en los cursos de Cálculo I, II y Ecuaciones Diferenciales en carreras de Ingeniería Civil. PU1 y PU2 dictan clases en la misma institución, mientras que PU3 dicta clases en otra institución universitaria.

En la entrevista se pregunta a cada profesor: “¿Cómo abordaría en sus clases el estudio de la sucesión

$\left(a_{n}\right) n \in \mathrm{N}$ ?". Para ello se les asignó un tiempo de una hora para desarrollar dicha pregunta por escrito. Sin embargo, al cabo de alrededor de 30 minutos, solicitaron más tiempo para enviar con posterioridad sus respuestas.

Cabe señalar que, en la enseñanza universitaria, según los programas y textos universitarios, y por la propia experiencia de los tres profesores entrevistados, quienes enseñan la unidad de sucesiones en el curso de Cálculo II, el estudio de la sucesión $\left(a_{n}\right) n \in \mathrm{N}$

en cuestión, se trata como un ejemplo clásico de aplicación del criterio de convergencia para sucesiones monótonas y acotadas.

\section{Estudio de los ETM idóneos de PU1, PU2 y PU3}

A continuación, analizaremos las producciones de los tres docentes universitarios respecto a la tarea que les fuera propuesta en la entrevista. En general, los tres profesores no generan tareas para abordar la sucesión, sino más bien ellos utilizan distintas herramientas para estudiarla, con el objetivo principal de probar que es convergente.

\subsection{ETM idóneo de PU1}

PU1 señaló que él haría preguntas a los estudiantes con la intención de guiar el problema solicitado. PU1 abordó la convergencia desde un punto de vista gráfico, proponiendo preguntas a los estudiantes (Figura 1) y dando las respuestas que este esperaría de parte de ellos (Figura 2).

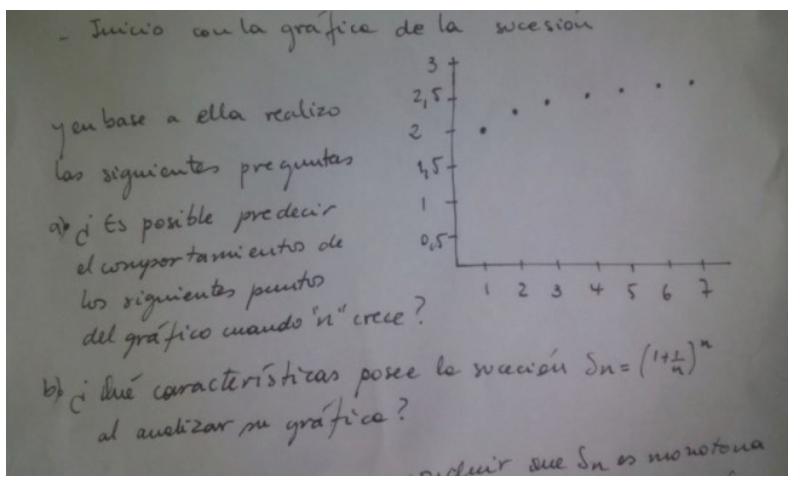

Transcripción:

Inicio con la gráfica de la sucesión y en base a ella realizo las siguientes preguntas:

a) ¿Es posible predecir el comportamiento de los siguientes puntos del gráfico cuando " $n$ " crece?

b) ¿Qué características posee la sucesión

$s_{n}=(1+1 / n)^{n}$ al analizar su gráfica?

Figura 1. Preguntas de PU1 para guiar el trabajo de los estudiantes.

Fuente: Verdugo-Hernández (2016, p. 97)

Según las respuestas que el propio profesor generada para las preguntas (a) y (b), se evidencia que el docente esperaría que los estudiantes, a partir de (a), deduzcan que la sucesión $s_{n}=n \in \mathrm{N} \quad$ es 
monótona creciente. De la pregunta (b), PU1 esperaría que los estudiantes utilicen la calculadora para deducir que la sucesión "tiende a aproximarse" a 2,72 y que posiblemente tracen la recta $y=2,72$, de modo que ellos visualicen que la sucesión es acotada superiormente por 2,72. En definitiva, PU1 pretende que sus estudiantes hagan una deducción basada en un análisis gráfico-numérico (realizando cálculos mediante una calculadora y eventualmente un gráfico), del hecho de que la sucesión es monótona creciente, acotada superiormente y convergente, relacionando así la convergencia más bien a la visualización, y no a los dos primeros aspectos que permitirían concluir teóricamente. En efecto, de acuerdo al criterio de convergencia de sucesiones monótonas y acotadas, herramienta teórica perteneciente al referencial de las sucesiones, del hecho de que la sucesión $s_{n}=n \in \mathrm{N}$ es monótona creciente y acotada (superiormente), se deduce que ella es convergente.

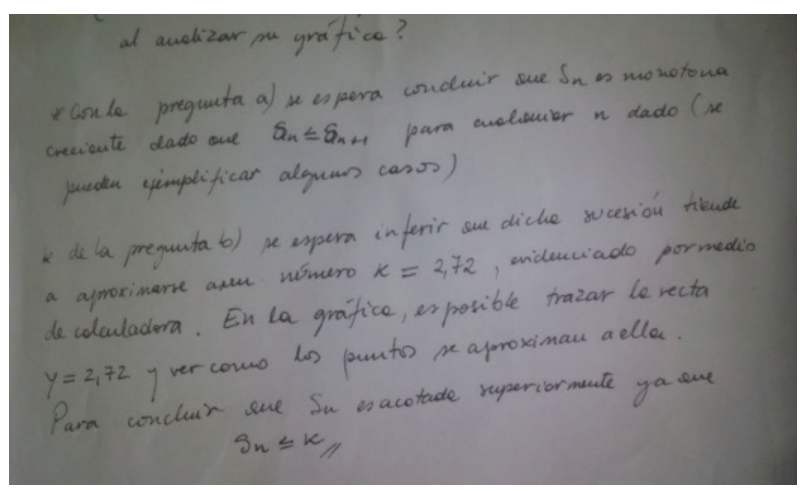

Transcripción:

* Con la pregunta a) se espera concluir que $S_{n}$ es monótona creciente dado que $s_{n} \leq s_{n}+1$ para cualquier $n$ dado (se puede ejemplificar algunos casos).

* De la pregunta b) se espera inferir que dicha sucesión tiende a aproximarse a un número

$k=2,72$, evidenciado por medio de calculadora. En la gráfica, es posible trazar la recta $y=2,72$, y ver cómo [sic] los puntos se aproximan a ella.

Para concluir que $\left(s_{n}\right) n \in N$ es acotada superiormente ya que $s_{n} \leq k$.

Figura 2. Respuesta entregada por PU1.

Fuente: Verdugo-Hernández (2016, p. 97)

En términos del ETM, PU1 activó la génesis semiótica e instrumental, pues el profesor insta a la comprensión de $\left(s_{n}\right) n \in N$ mediante un gráfico, mostrando que la sucesión es monótona creciente, lo cual genera el plano vertical [Sem-Ins]. Como artefacto se identifica el uso de la calculadora, y en términos de signos y registros el tratamiento es gráfico.

Por otro lado, como ya vimos, la producción de PU1 está basada en un análisis gráfico-numérico, ya que se evidencia que este esperaba la utilización de una calculadora para que los estudiantes obtuvieran una aproximación del límite (el cual sabemos que es el número irracional $e$ ), y que eventualmente trazaran una recta $(y=2,72)$, de modo tal de visualizar que la sucesión es acotada superiormente. Todo lo anterior nos indica que el trabajo de PU1 se enmarca en el paradigma Análisis-Geométrico/Aritmético (AG) (Montoya y Vivier, 2016), que permite realizar interpretaciones nacidas de la geometría y/o del cálculo aritmético, lo cual se ajusta a la actividad realizada por PU1.

\subsection{ETM idóneo de PU2}

PU2 privilegia el uso de la regla de L'Hôpital, herramienta teórica perteneciente al referencial de las funciones, con la cual es posible calcular el límite de la sucesión aplicando un resultado previo que conecta las sucesiones con las funciones. Volveremos sobre este punto más adelante.

Tal como mencionamos en la introducción, el análisis de convergencia de la sucesión $\left(a_{n}\right) n \in \mathrm{N}$ puede ser llevado a cabo mediante el criterio de convergencia de sucesiones monótonas y acotadas, que establece que todasucesióncreciente(respectivamente, decreciente) y acotada superiormente (respectivamente, acotada inferiormente) es convergente. Dado que PU2 aplicó la regla de L'Hôpital en lugar de utilizar el criterio de convergencia mencionado, y como este docente fue el único en entregar una respuesta en el mismo momento en que se le entrevistó, tuvimos la oportunidad de preguntarle, justo después de entregar su respuesta, las razones por las cuales utilizó dicha herramienta. PU2 responde que "se debe a que los estudiantes están más familiarizados con esta herramienta, debido a que en el curso previo la utilizaron bastante".

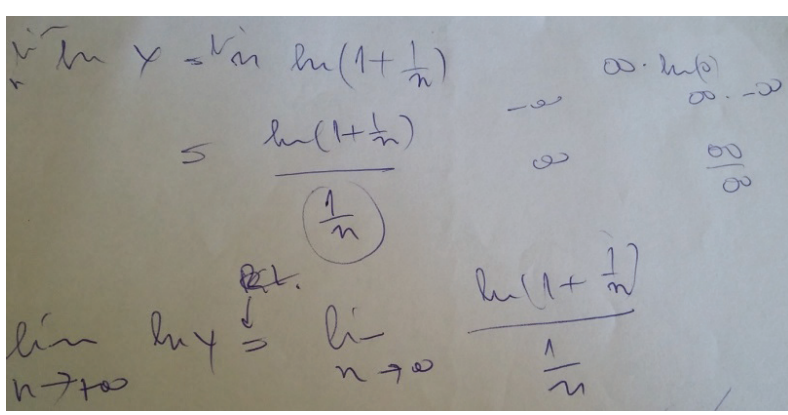

Figura 3. Respuesta de PU2

Fuente: Verdugo-Hernández (2016, p. 98)

En las Figuras 3 y 4 se puede observar que PU2 no mencionó que para su desarrollo es necesario pasar del cálculo del límite de la sucesión $\left(a_{n}\right) n \in \mathrm{N}$ a un límite de una función de una variable real, con el fin de aplicar la regla de L'Hôpital, y enseguida devolverse 
del límite de esta función para obtener el valor del límite de la sucesión $\left(a_{n}\right) n \in \mathrm{N}$. Con el fin de que su cálculo sea válido, basta aplicar el resultado previo siguiente: Si $\lim _{x \rightarrow \infty} f(x)=L$ y $f(n)=a_{n}$, cuando es un entero, entonces $\lim _{x \rightarrow \infty} a_{n}=L^{1}$. Esta herramienta teórica conecta ambos objetos matemáticos, sucesiones y funciones, y sobre todo provee una forma sencilla de calcular límites de sucesiones, por medio del cálculo de límites de funciones al infinito (cuando la variable $x$ tiende al infinito). Cabe notar que, en este caso, la función $f(x)$ posee una asíntota horizontal $y=L^{2}$.

Notemos que, si deseamos aplicar la regla de L'Hôpital, herramienta teórica para calcular límites de funciones, es necesario previamente establecer una conexión entre ambos objetos matemáticos, tal como hemos planteado en el párrafo anterior. Para ser más precisos, de la sucesión $f(n)=a_{n}$ pasamos a la función $x \rightarrow f(x)$, lo cual implica un paso de lo discreto a lo continuo, relacionando ambos conceptos. Al respecto, PU2 no hace explícitamente esta diferencia, ya que este no toma en cuenta la dialéctica discreta/continuo. Sería interesante estudiar qué consecuencias podría esto traer para los estudiantes, lo cual es materia de otro trabajo.

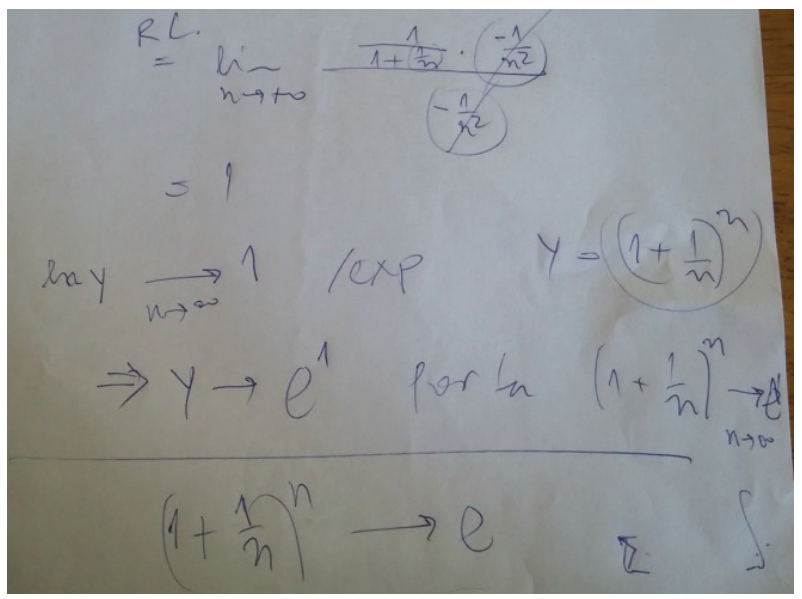

Figura 4. Propuesta de PU2 (continuación). Fuente: Verdugo-Hernández (2016, p. 99)

En los cálculos realizados (Figura 4), PU2 da por hecho que el número e existe cuando calcula el límite. Sin embargo, el número $e$ existe porque se le puede definir como el límite de la sucesión analizada, es decir, lo que hace PU2 es utilizar precisamente lo que hay que probar que existe (el límite de la sucesión analizada en este trabajo). Existen además otras formas de definir el número $e$ (por ejemplo, definir la función logaritmo natural por medio de una integral definida, y enseguida definir el número $e$ como la base de dicho logaritmo), pero la idea central que deseamos rescatar es que, al menos en el nivel universitario, hay que definir tal número antes de proceder a utilizarlo.

Por otro lado, tal como fue mencionado anteriormente, PU2 calculó el límite de la sucesión aplicando la regla de L'Hôpital, en lugar de aplicar el criterio de sucesiones monótonas y acotadas, por la razón que el mismo PU2 señaló. Para utilizar el criterio mencionado, PU2 debería haber probado que la sucesión $\left(a_{n}\right) n \in \mathrm{N}$ es monótona y acotada (en este caso, se puede probar que $\left(a_{n}\right) n \in \mathrm{N} \quad$ es creciente y acotada superiormente). De hecho, con el fin de abordar los aspectos previos, PU2 propuso espontáneamente las siguientes indicaciones (sin demostración), las cuales él dejaría a cargo de los estudiantes:

(1) Probar que $\left(a_{n}\right) n \in \mathrm{N}$ es creciente usando el teorema del binomio y que $1-i / n<1-i /(n+1)$ para cada $i=1,2, \ldots, n .-1$.

(2) Para demostrar que $\left(a_{n}\right) n \in \mathrm{N}$ es acotada usar que $i !>2 i-1$, para cada $i \in N$ (mostrar la desigualdad por inducción).

Desde nuestro marco, se observa que PU2 utilizó una herramienta de las funciones de variable real (la regla de L'Hôpital) con el objetivo de calcular el límite. Luego, sugiere implícitamente (pues no lo menciona) utilizar el criterio de las sucesiones monótonas y acotadas, para lo cual propuso los puntos (1) y (2), los cuales quedan completamente dentro del referencial de las sucesiones (sin recurrir a herramientas pertenecientes a las funciones), con el fin de probar que $\left(a_{n}\right) n \in \mathrm{N}$ es creciente y acotada superiormente. Consideramos que en esta propuesta

\footnotetext{
${ }^{1}$ Para un mayor detalle, remitirse teorema 3 presente en el texto de J. Stewart (2012, p. 678).

${ }^{2}$ Ver Figura 6 (Stewart, 2012, p. 678).

${ }^{3}$ Suponemos que PU2 propone las indicaciones transcritas debido a que considera importante probar la monotonía y el acotamiento de (an), ya que posiblemente cree que el cálculo del límite con la regla de L'Hôpital es insuficiente.
} 
se activaron la génesis instrumental, en el sentido de las herramientas utilizadas (artefactos teóricos), y la génesis semiótica, activando el plano vertical [SemIns]. Además, se induce la activación del plano vertical [Sem-Dis] en el momento que PU2 deja propuestos los puntos (1) y (2), invitando a los estudiantes a realizar dichas pruebas.

Por último, la propuesta se centra en el paradigma de Análisis-Calculatorio (AC), puesto que utiliza reglas explícitas del cálculo (la regla de L'Hôpital), sin cuestionarse la naturaleza de los objetos matemáticos involucrados, ya que, de hecho, desde el problema original que consiste en analizar una sucesión, pasa implícitamente a estudiar el límite de una función, sin cuestionarse ni el paso ni el nuevo objeto introducido (la función). En este sentido, notemos que hay aspectos importantes que subyacen al paso de lo discreto a lo continuo. En efecto, para diferenciar adecuadamente ambos conceptos (discreto y continuo), hay que tener en cuenta la completitud, ya que "lo continuo" hace referencia en realidad a aquello que "no tiene agujeros". Esa sutil diferencia constituye el por qué las sucesiones son tratadas de manera especial y no como funciones ordinarias de variable real, aspecto que pensamos que merece ser bien estudiado en clases por parte de los docentes.

\subsection{ETM idóneo de PU3}

A continuación, mostraremos la producción del docente PU3. Preliminarmente, PU3 señaló que aplicaría algunas herramientas de cursos previos tales como el teorema del binomio, el que, si bien es cierto que en términos del ETM en otro curso podría ser parte del referencial teórico, en este, donde se estudian sucesiones, deviene una herramienta operatoria (Verdugo-Hernández, 2018) que permite comprobar que la sucesión analizada es acotada.

PU3: Conocidas algunas definiciones y desigualdades básicas en los cursos de Matemática, como son el teorema del binomio, ser acotado y la monotonía de una sucesión, por el teorema del binomio, se tiene que:

$$
\begin{aligned}
& a_{n}=1+\left(\frac{1}{n}\right) n=\sum_{k}^{n}=0\left(\begin{array}{l}
n \\
k
\end{array}\right)\left(\frac{1}{n}\right)=1+n \cdot \frac{1}{n}+\frac{n(n+1)}{2 ;} \cdot \frac{1}{n^{2}}+\ldots+\frac{n(n-1)}{n ;} \frac{1}{n^{n}} \\
& =1+1+\frac{1}{2}\left(1-\frac{1}{n}\right)+\frac{1}{3}\left(1-\frac{1}{n}\right) \cdot\left(1+\frac{1}{2}\right)+\ldots+\frac{1}{n}\left(1-\frac{1}{n}\right) \cdot\left(1-\frac{2}{n}\right) \cdot \ldots \cdot\left(1-\frac{n-1}{n}\right)
\end{aligned}
$$

A continuación, PU3 introdujo tres sucesiones $\left(a_{n}\right) n \in \mathrm{N},\left(b_{n}\right) n \in \mathrm{N}$ y $\left(c_{n}\right) n \in \mathrm{N}$. Este sugirió entonces comparar término a término dichas sucesiones a partir de $n=3$, con el fin de concluir que la sucesión $\left(a_{n}\right) n \in \mathrm{N}$ es acotada.

PU3: Se comprueba que la sucesión está acotada, para lo cual se consideran las siguientes expresiones:

$$
\begin{aligned}
& =2+\frac{1}{2 ;}\left(1-\frac{1}{n}\right)+\frac{1}{3 ;}\left(1-\frac{1}{n}\right) \cdot\left(1-\frac{1}{2}\right)+\ldots+\frac{1}{n}\left(1-\frac{1}{n}\right)\left(1-\frac{2}{n}\right) \ldots\left(1-\frac{1}{n}\right) \\
& =2+\frac{1}{2 ;}+\frac{1}{3}+\ldots+\frac{1}{;} \\
& =2+\underbrace{\frac{1}{2}+\frac{1}{2}+\ldots+\frac{1}{2^{n-1}}}_{\text {progresión geométrica }}=3-\frac{1}{2^{n-1}}
\end{aligned}
$$

En efecto, comparando término a término a partir de $n=3$ se verifica que: $2<a_{n}<b_{n}<c_{n}=3-1 / 2^{n-1}$, de donde $2<a_{n}<3$; luego, la sucesión $\left(a_{n}\right) n \in \mathrm{N}$ es acotada.

Ahora se demuestra que la sucesión es estrictamente creciente de la siguiente manera:

$M . A>M . G \Leftrightarrow \frac{\left(1+\frac{1}{n}\right)+\left(1+\frac{1}{n}\right)+\ldots+\left(1+\frac{1}{n}\right)+1}{n+1}>n+1 \sqrt{\left(1+\frac{1}{n}\right)^{n}}$

$\Leftrightarrow n \cdot \frac{\left(1+\frac{1}{n}\right)+1}{n+1}>\sqrt[n+1]{\left(1+\frac{1}{n}\right)^{n}}$

$\Leftrightarrow\left(1+\frac{1}{n+1}\right)^{n+1}>\left(1+\frac{1}{n}\right)^{n}$

$\Leftrightarrow a_{n+1}>a_{n}$ Luego, $\left(a_{n}\right) n \in \mathrm{N}$ es monótona creciente.

Así PU3 concluyó, considerando los dos aspectos anteriores (acotamiento y monotonía) que $\left(a_{n}\right) n \in \mathrm{N}$ es convergente. Además, señaló que, en consecuencia, "tiene límite finito". Ese límite es precisamente el número $e$, irracional y trascendente, la demostración anterior activa la génesis discursiva. Para visualizar este resultado el docente consideró el siguiente gráfico (Figura 5) que indica el comportamiento de la sucesión, convergiendo al número $e$, activando la génesis instrumental. PU3 indica que "el comportamiento de convergencia hacia el valor de e por medio del gráfico es lento", lo cual significa que 
numéricamente la sucesión se demora en acercarse hacia la recta $y=e$ (en el gráfico aún no se aprecia claramente que los puntos se acerquen a la recta $y=e$; creemos que hubiese hecho falta graficar más puntos de la sucesión para acercarse de mejor forma).

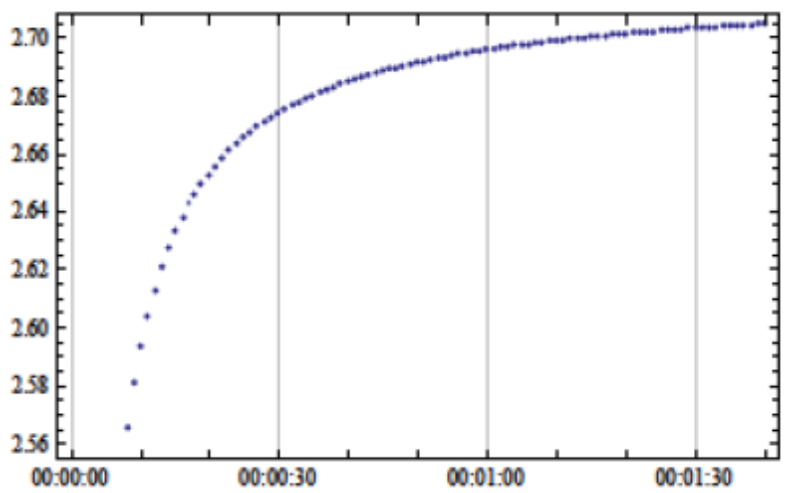

Figura 5. Gráfico de la sucesión $\left(a_{n}\right) n \in \mathrm{N}$ Fuente: Verdugo-Hernández (2016, p. 101)

En términos del ETM, PU3 realizó una propuesta en donde se activaron las génesis discursiva e instrumental, evidenciando la activación de los planos verticales [Sem-Dis] y [Sem-Ins]. El plano vertical [Sem-Ins] se evidencia debido a que el docente activa la génesis instrumental a través del gráfico realizado por medio del software Maple. El plano vertical [Sem-Dis] se activa debido a que el docente activa la génesis discursiva en dirección de la prueba, ya que demuestra el acotamiento y la monotonía de la sucesión $\left(a_{n}\right) n \in \mathrm{N}$.

Además, se realiza una segunda entrevista a PU3 para consultarle si él genera regularmente actividades con Maple en sus clases, a lo cual él afirma que sí, aunque dicha información no se pudo corroborar.

Se puede constatar que el trabajo matemático desarrollado por el docente se encuentra en el paradigma $\mathrm{AC}$, ya que este utiliza reglas del cálculo, sin necesariamente profundizar en la naturaleza de los objetos. Podemos señalar además que la propuesta de PU3 posee un razonamiento propio del dominio específico de las sucesiones, dado que utiliza herramientas relacionadas con estas, demostrando detalladamente el acotamiento y la monotonía. La importancia de estos aspectos radica en la utilización del referencial propio de las sucesiones, ya que, por lo dicho anteriormente, una de las herramientas fundamentales para el estudio de la convergencia de las sucesiones es el criterio de convergencia de las sucesiones monótonas y acotadas. Entonces, de hecho, para aplicar este criterio, ni siquiera se necesita calcular el límite (el cual se debería calcular una vez que se sabe que la sucesión es convergente, si es posible), sino que basta con probar la monotonía y el acotamiento para demostrar la convergencia. Por esa razón es una herramienta teórica importante, ya que ni siquiera se requiere conocer explícitamente el término general de una sucesión para probar que es convergente.

\section{Conclusiones}

En esta investigación hemos realizado un estudio de caso para abordar las posibles formas de enseñanza de la sucesión $\left(a_{n}\right) n \in \mathrm{N}$, por medio del análisis de las producciones de tres docentes universitarios en relación a cómo ellos tratarían esta cuestión frente a sus eventuales estudiantes. Para esto, hemos aplicado el modelo del ETM del análisis real, analizando particularmente el ETM idóneo universitario, considerando las distintas componentes, génesis y planos, así como también los distintos paradigmas del análisis real.

Desde el punto de vista teórico, hemos analizado el ETM idóneo, a partir de lo cual evidenciamos que PU1 trabaja mucho más en el desarrollo de la génesis semiótica en su propuesta, mientras que los otros dos profesores universitarios abordan lo solicitado activando también las otras dos génesis. En concordancia con lo anterior, los tres docentes activan distintos planos verticales, evidenciando que PU2 y PU3 privilegian por sobre todo la prueba. Sin embargo, esto podría obedecer a distintas razones, entre las cuales estaría el tipo de carrera hacia donde están dirigidas las clases, dado que PU2 y PU3 enseñan en carreras de Ingeniería Civil, donde supuestamente la complejidad matemática debería ser mayor, mientras que PU1 realiza clases en Ingeniera Comercial, donde la complejidad apuntaría a otras áreas del conocimiento.

En relación a los paradigmas, constatamos que el trabajo de estos tres profesores universitarios se posiciona en los paradigmas AG y AC. Debemos señalar que en este estudio de caso, en donde nos centramos solamente en la producción de los docentes relativa al tratamiento de la sucesión $\left(a_{n}\right) n \in \mathrm{N}$, no evidenciamos el paradigma $A R$, cuestión que era esperable, ya que para lo anterior requeririamos considerar otros elementos que contribuyan al ETM idóneo, tales como el estudio teórico de las sucesiones, donde se introduzca al menos la noción de convergencia, involucrando la completitud del sistema de los números reales. Asimismo, a pesar de que los ETM idóneos estudiados se enmarcan, como ya hemos mencionado, en distintos paradigmas, destacamos que el artefacto (software en este caso) podría jugar un rol importante en la estructuración del ETM idóneo. Por otro lado, es interesante destacar un tipo de trabajo que privilegia la utilización de herramientas teóricas de las funciones (la regla de L'Hôpital), y otro tipo que no las incluye, pero que incorpora herramientas operatorias, las cuales pertenecen a otros dominios de las matemáticas (el teorema del binomio), dando cuenta de dos líneas de trabajo bien diferenciadas en relación a las sucesiones. 
En este trabajo se ha constatado que las propuestas de los docentes privilegian distintas componentes de trabajo en el ETM. En principio no se puede confirmar que alguna de estas propuestas sea mejor que las otras, sin embargo, el estudio del ETM nos indica claramente que algunos docentes han desarrollado más ciertas componentes que otras. En este sentido postulamos que, abarcando una mayor cantidad de génesis y componentes, y generando una mayor circulación entre ellas, es decir, activando los distintos planos verticales, se estaría ofreciendo la posibilidad de una mejor enseñanza y comprensión de las materias. Asimismo, en concordancia con lo anterior, pensamos que el hecho de estructurar la enseñanza basada en los tres paradigmas podría apoyar la concepción de un posible trabajo matemático completo, en el sentido de presentar una propuesta que abarque la integración coherente de ellos, dando cabida a un trabajo de enseñanza más amplio e incorporando un proceso completo en el desarrollo del trabajo del docente.

En consecuencia, creemos que es interesante que los profesores enseñen posicionándose en los tres paradigmas y puedan mostrar las limitaciones y ventajas de usar uno por sobre el otro, y sobre todo las ventajas de integrarlos holísticamente de forma coherente. En este marco de trabajo, sería deseable obtener resultados desde el punto de vista del ETM personal del estudiante, en donde los docentes apliquen un ETM idóneo transitando entre los paradigmas y no solamente privilegiando uno sobre otro. En ese sentido, sería adecuado integrar el aspecto gráfico de las sucesiones con el aspecto teórico, sin descuidar este último, de manera de producir una mayor comprensión en los estudiantes sobre las tareas teóricas que se esperaría que ellos enfrentaran, algunas de las cuales sintetizamos en este trabajo por medio de la sucesión estudiada a través de las producciones de los docentes universitarios. En todo caso, queda abierto analizar si efectivamente los estudiantes lograrían apropiarse más de los conceptos teóricos si se incorporaran los aspectos gráficos de las sucesiones, y asimismo si se integraran todos los paradigmas del análisis, incluyendo los planos verticales y génesis del ETM, importante cuestión que será analizada en otro trabajo.

\section{Agradecimiento:}

Proyectos de Investigación Regular (2019-113). Universidad Adventista de Chile. 


\section{Referencias bibliográficas}

Artigue, M. (1998). Enseñanza y aprendizaje del análisis elemental: ¿qué se puede aprender de las investigaciones didácticas y los cambios curriculares? Revista Latinoamericana de Investigación en Matemática Educativa, 1(1), 40-55. Recuperado desde http://funes. uniandes.edu.co/9584/1/Artigue1998Enseñanza.pdf

Bergé, A. (2008). The completeness property of the set of real numbers in the transition from calculus to analysis. Educational Studies in Mathematics, 67(3), 217235. https://doi.org/10.1007/s10649-007-9101-5

Bloch, I. (2000). L'enseignement de l'analyse à la charnière lycée/université. Savoirs, connaissances et conditions relatvies à la validation (Tesis doctoral). Université Bordeaux I, Burdeos, Francia. Recuperado desde https://hal.archives-ouvertes.fr/tel-01222400/ document

Bobadilla, G., y Labarca, R. (2004). Cálculo. Santiago: Facultad de Ciencias. Universidad de Santiago de Chile.

Dhombres, J. (1980). Nombre, mesure et continu: Epistémologie et histoire. París: CEDIC.

Gómez-Chacón, I. M., Kuzniak, A., y Vivier, L. (2016). El rol del profesor desde la perspectiva de los Espacios de Trabajo Matemático. Bolema, 30(54), 1-22. https:// doi.org/10.1590/1980-4415v30n54a01

Hairer, E., y Wanner, G. (2000). L'analyse au fil de I'histoire. Alemania: Springer-Verlag.

Kuratowski, K. (1962). Introduction to calculus. Massachusetts, U.S.A.: Addison-Wesley Publishing Company Inc. Reading.

Kuzniak, A. (2011). L'Espace de Travail Mathematique et ses genèses. Annales de Didactique et de Sciences Cognitives, 6, 9-24. Recuperado desde https://halshs. archives-ouvertes.fr/halshs-01060043/document

Kuzniak, A., Montoya-Delgadillo, E., y Vivier, L. (2016). El espacio de trabajo matemático y sus génesis. Cuadernos de Investigación y Formación en Educación Matemática, 5, 237-251. Recuperado desde https:// revistas.ucr.ac.cr/index.php/cifem/article/view/23942

Kuzniak, A., y Richard, P. (2014). Spaces for mathematical work: Viewpoints and perspectives. Relime, 17(4.1), 17-26. https://doi.org/10.12802/ relime.13.1741b

Mamona-Downs, J. (2001). Letting the intuitive bear on the formal; a didactical approach for the understanding of the limit of a sequence. Educational Studies in Mathematics, 48(2-3), 259-288. https://doi. org/10.1023/A:1016004822476
Montoya, E., y Vivier, L. (2016). Mathematical Working Spaces as an analyzing tool for the teaching and learning of calculus. ZDM, 48(6), 739-754. https://doi. org/10.1007/s11858-016-0777-9

Oktaç, A., y Vivier, L. (2016). Conversion, Change, Trnsition... in Research About Analysisi. En B. Hodgson, A. Kuzniak, y J. B. Lagrange (Eds.), The Didactics of Mathematics: Approaches and Issues (pp. 87-121). New York: Springer. https://doi.org/10.1007/978-3-31926047-1_5

Robert, A. (1982). Acquisition de la notion de convergence des suites numériques dans l'enseignement supérieur (Tesis doctoral). Université de Paris VII, París, Francia. Recuperado desde https://tel.archives-ouvertes.fr/tel01250393/file/thèse\%2ORobert.pdf

Stewart, J. (2012). Cálculo de una variable. México: Prentice Hall Hispanoamérica.

Verdugo-Hernández, P. (2018). Espacio de Trabajo Matemático del Análisis: Enseñanza de las sucesiones en los primeros años de universidad (Tesis doctoral). Pontificia Universidad Católica de Valparaíso, Valparaíso, Chile. Recuperado desde http://repositorio. conicyt.cl/bitstream/handle/10533/208871/ANEXOS. pdf? sequence $=4$

Verdugo- Hernández, P. (2016). Una Aproximación al ETM idóneo de las Sucesiones: el caso de la Sucesión $(1+1 / n) n$ en la Enseñanza Secundaria y Universitaria. En I. Gómez-Chacón, A. Kuzniak, K. Nikolantonakis, R. Richard y L. Vivier (Eds.), Acta del Quinto Simposio Internacional ETM (pp. 95-103). Florina, Grecia. 